Published in final edited form as:

Nat Nanotechnol. 2020 January ; 15(1): 41-46. doi:10.1038/s41565-019-0600-1.

\title{
Vitamin Lipid Nanoparticles Enable Adoptive Macrophage Transfer for the Treatment of Multidrug-Resistant Bacterial Sepsis
}

\author{
Xucheng Hou ${ }^{\# 1}$, Xinfu Zhang ${ }^{\# 1,2}$, Weiyu Zhao ${ }^{1}$, Chunxi Zeng ${ }^{1}$, Binbin Deng ${ }^{3}$, David W. \\ McComb $^{3,4}$, Shi Du ${ }^{1}$, Chengxiang Zhang ${ }^{1}$, Wenqing Li ${ }^{1}$, Yizhou Dong ${ }^{1,5},{ }^{*}$ \\ ${ }^{1}$ Division of Pharmaceutics \& Pharmacology, College of Pharmacy, The Ohio State University, \\ Columbus, Ohio 43210, USA. \\ ${ }^{2}$ State Key Laboratory of Fine Chemicals, Dalian University of Technology, Dalian 116024, China. \\ ${ }^{3}$ Center for Electron Microscopy and Analysis, The Ohio State University, Columbus, $\mathrm{OH} 43212$, \\ USA \\ ${ }^{4}$ Department of Materials Science and Engineering, The Ohio State University, Columbus, $\mathrm{OH}$ \\ 43210, USA \\ ${ }^{5}$ Department of Biomedical Engineering, Center for Clinical and Translational Science, \\ Comprehensive Cancer Center, Dorothy M. Davis Heart \& Lung Research Institute, Department \\ of Radiation Oncology, The Ohio State University, Columbus, Ohio 43210, USA. \\ \# These authors contributed equally to this work.
}

\begin{abstract}
Sepsis, a condition caused by severe infections, affects more than 30 million people worldwide every year and remains the leading cause of death in hospitals ${ }^{1,2}$. Moreover, antimicrobial resistance has become an additional challenge in the treatment of sepsis ${ }^{3}$, and thus, alternative therapeutic approaches are urgently needed ${ }^{2,3}$. Here, we show that adoptive transfer of macrophages containing antimicrobial peptides linked to cathepsin B in the lysosomes (MACs) can be applied for the treatment of multi-drug resistant (MDR) bacteria-induced sepsis in mice with immunosuppression. The MACs are constructed by transfection of vitamin $\mathrm{C}$ lipid nanoparticles $\left(\mathrm{V}_{\mathrm{C}} \mathrm{LNPs}\right)$ that deliver antimicrobial peptide and cathepsin B (AMP-CatB) mRNA.
\end{abstract}

\footnotetext{
Reprints and permission information is available online at www.nature.com/reprints.

*Correspondence and requests for materials should be addressed to Y.D., dong.525@ osu.edu. Author contributions

X.H. and X.Z. conceived and designed the experiments. X.H. and X.Z. performed the experiments and wrote the paper. W.Z. contributed to animal and live-cell imaging. C.Zeng. prepared mRNA. B.D. and D.W.M. contributed to Cryo-TEM imaging. S.D. and W.L. contributed to animal experiments. C.Zhang contributed to flow cytometry assays. Y.D. conceived and supervised the project, and wrote the paper. The final manuscript was edited and approved by all authors.

Competing interests

The authors have no competing interests to declare.

Supplementary information is available in the online version of the paper.

Data availability

The data that support the plots within this paper and other findings of this study are available from the corresponding author upon reasonable request.
} 
The $\mathrm{V}_{\mathrm{C}} \mathrm{LNPs}$ allow the specific accumulation of AMP-CatB in macrophage lysosomes, which is the key location for bactericidal activities. Our results demonstrate that adoptive MAC transfer leads to the elimination of MDR bacteria, including Staphylococcus aureus and Escherichia coli, leading to the complete recovery of immunocompromised septic mice. Our work provides an alternative strategy for overcoming MDR bacteria-induced sepsis and opens up possibilities for the development of nanoparticle-enabled cell therapy for infectious diseases.

Traditionally, sepsis has been viewed as an uncontrollable inflammatory response to pathogens ${ }^{2,4}$. However, recent clinical data reveal that more than $60 \%$ of septic patients survive through the initial inflammatory storm but rapidly progress to a longer immunosuppressive state, which is characterized by the paralysis and death of immune cells, leading to an inability to clear invading pathogens, increased susceptibility to hospitalacquired infections, and high mortality rate ${ }^{2,4-6}$. As a result, potential therapeutic targets have been extensively explored in order to treat sepsis such as removing the anaphylatoxin C5a or blocking the C5a receptor ${ }^{7,8}$. Meanwhile, approaches aiming to restore immune function have been developed and tested in patients with sepsis ${ }^{2,4,6}$. Macrophages are one of the most efficient pathogen scavengers during infections ${ }^{9}$. Indeed, in septic patients, impaired macrophages/monocytes seem to be primarily responsible for insufficient antimicrobial defense ${ }^{6,9-12}$. Several small clinical trials of immunostimulatory agents indicate benefits in reversing the deactivated macrophages/monocytes, thus enhancing infection eradication ${ }^{10,11}$. On the other hand, meta-analysis of large clinical trials did not show significant changes in reducing the patient mortality ${ }^{13}$. Differences in clinical results may be explained as follows ${ }^{10,14-16}$. First, immunostimulatory agents are not able to restore the function of impaired macrophages/monocytes to their original levels ${ }^{10}$. Second, invaded bacteria are usually trapped in macrophage phagolysosomes, where reactive oxygen species, reactive nitrogen species, and lysozymes work synergistically to clear bacteria ${ }^{17}$. However, many bacteria, such as Staphylococcus aureus and Escherichia coli, have evolved immune escape mechanisms for thwarting phagolysosomal killing, resulting in intracellular survival and recurrent infections ${ }^{14-16}$. Third, although antibiotic therapy is the standard treatment in the sepsis clinical guideline, $70 \%-80 \%$ of sepsis death is related to persistent infections, thus indicating the prevalence of antibiotic resistance and the paucity of effective antimicrobial agents $^{3,6}$.

Based on the above research findings, we hypothesized that adoptive transfer of macrophages containing antimicrobial peptides linked to cathepsin B in the lysosomes (MACs) may enable the immunocompromised sepsis host to boost innate immunity, prevent bacterial immune evasion, and eliminate multi-drug resistant (MDR) bacteria. To test our hypothesis, we first designed and constructed the AMP-CatB mRNA encoding an antimicrobial peptide IB367 (AMP-IB367), a cathepsin B (CatB), and a CatB-sensitive linker. AMP-IB367 is a broad-spectrum AMP with rapid bactericidal activity, and has been confirmed in clinical trials ${ }^{18}$. CatB, an endogenous lysosomal protein, is first translated as an inactive precursor in the cytoplasm. After translocation into the lysosome, the precursor is processed into mature $\mathrm{CatB}^{19}$. In this study, the $\mathrm{CatB}$ component is incorporated to transport the AMP-IB367 into lysosomes. In order to equip macrophages with the AMP-CatB mRNA, we designed a series of vitamin-derived lipid nanomaterials. As illustrated in Fig. 1a, the 
mRNA is translated to functional proteins in the cytoplasm and the proteins are further translocated into lysosomes ${ }^{19}$, where the linker is cleaved by CatB proteins and thus the APM-IB367 is released ${ }^{20}$. When phagosomes encapsulating bacteria fuse with lysosomes, the ingested bacteria are exposed to both pre-stored AMP-IB367 and lysosomal antimicrobial components. Although immune evasion strategies may protect MDR bacteria from phagolysosomal killing mechanisms, the AMP-IB367 is able to kill these bacteria. Consequently, the adoptive transfer of MACs rescue MDR bacteria-induced sepsis mice with immunosuppression by restoring the innate immunity, overcoming bacterial immune evasion, and eradicating the infection.

In this study, we first synthesized five vitamin-derived lipids: $\mathrm{V}_{\mathrm{B} 3}$-Lipid, $\mathrm{V}_{\mathrm{C}^{-}}$-Lipid, $\mathrm{V}_{\mathrm{D}^{-}}$

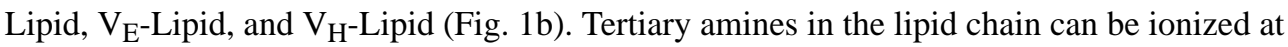
an acidic condition and interact with mRNAs. The structures of these vitamin-derived lipids were confirmed by ${ }^{1} \mathrm{H}$ NMR and mass spectrum (Supplementary Fig. 1-5).

Next, we formulated these vitamin-derived lipids into vitamin lipid nanoparticles (VLNPs) accordingly. Particle size of VLNPs ranged from $127 \pm 1$ to $174 \pm 1 \mathrm{~nm}$ with polydispersity index (PDI) $<0.3$ except $\mathrm{V}_{\mathrm{H}}$ LNPs (Supplementary Fig. 6a, b). Entrapment efficiency of mRNA was from $52 \%$ to $99 \%$ and all VLNPs were positively charged (Supplementary Fig. 6c, d). In initial screening in RAW264.7 cells using mRNA encoding firefly luciferase, we noted that $\mathrm{V}_{\mathrm{C}} \mathrm{LNPs}$ were 20 -fold more effective for mRNA delivery than other four VLNPs (Fig. 2a). Moreover, $V_{C} L N P s$ were 10 -fold better than Lipofectamine 3000 and were 50-fold better than electroporation at the same mRNA concentration (Fig. 2a). In addition, the highest luminescence intensity of $\mathrm{V}_{\mathrm{C}} \mathrm{LNP}$ group was observed at $12 \mathrm{~h}$ from the 6 to $24 \mathrm{~h}$ time course (Fig. 2b). To further optimize the formulation of $\mathrm{V}_{\mathrm{C}} \mathrm{LNPs}$, we prepared 16 different formulations based on an $\mathrm{L}_{16}(4)^{4}$ orthogonal table (Supplementary Fig. 6e). Formulation B2 (Lipid:DOPE:Cholesterol = 30:30:40) emerged from this study (Fig. 2c, d). Then, we validated its delivery efficiency by comparison with the top formulation A10 (Lipid:DOPE:Cholesterol = 30:30:50) in the orthogonal table. Luminescence intensity of the predicted formulation B2 was significantly higher than formulation A10 $(P<0.05$, Fig. 2 d, e). In the second round of optimization, we examined the mass ratio of $\mathrm{V}_{\mathrm{C}}$-Lipid:mRNA of formulation B2 from 5:1 to 20:1 (Fig. 2d). Luminescence intensity was enhanced as we increased the mass ratio of $\mathrm{V}_{\mathrm{C}}$-Lipid:mRNA to 15:1 (formulation C5, Fig. 2d, e).

Formulation $\mathrm{C} 5$ of $\mathrm{V}_{\mathrm{C}} \mathrm{LNPs}$ was in a spherical morphology from the Cryo-TEM image (Fig. $2 f$, Supplementary Fig. 6f). Based on these results, we chose this $V_{C} L N P s$ formulation with improved mRNA delivery efficiency over 7-fold than its initial formulation for further studies including cellular uptake, endocytic pathways, and endosomal escape. Using an Alexa-Fluor 647 labelled RNA, we observed more efficient cellular uptake of $\mathrm{V}_{\mathrm{C}} \mathrm{LNPs}$ than Lipofectamine 3000 and electroporation (Supplementary Fig. 7a, b). After incubation with methyl-beta-cyclodextrin $(\mathrm{M} \beta \mathrm{CD}$, inhibitor of caveolae-mediated endocytosis), cellular uptake of $V_{C} L N P s$ was dramatically reduced by about $96 \%$ (Supplementary Fig. 7c, d), indicating a major role of caveolae-mediated endocytosis for these $\mathrm{V}_{\mathrm{C}} \mathrm{LNPs}$. To explore the endosomal escape mechanism, we performed a calcein assay. Calcein, a membraneimpermeable dye, is normally entrapped into cell endosomes. We treated cells with both calcein and $\mathrm{V}_{\mathrm{C}} \mathrm{LNPs}$, and observed diffused green fluorescence in cell cytoplasm, 
suggesting that endosome membranes were ruptured and consequently $\mathrm{V}_{\mathrm{C}} \mathrm{LNPs}$ are released to the cytoplasm (Supplementary Fig. 7e, f).

To test whether CatB was able to transport the payload into the lysosomes, we constructed eGFP-CatB mRNA and delivered it into RAW264.7 cells using V $\mathrm{V}_{\mathrm{C}} \mathrm{LNPs}$. Confocal images of live cells exhibited that eGFP-CatB co-localized with LysoTracker® Red DND-99 in lysosomes, with a Pearson's correlation coefficient of 0.93 (Fig. 2g, Supplementary Fig. 7g), indicating that CatB carried its payload into lysosomes. Then, to evaluate the bactericidal activity of MACs, we quantified the intracellular survival of multi-drug resistant Staphylococcus aureus (MDRSA) in RAW264.7 cells treated by PBS (PBS-RAWs), free AMP-CatB mRNA (Fr-RAWs), empty $V_{C}$ LNPs (Em-RAWs), AMP-CatB mRNA $V_{C} L N P s /$ CatB inhibitor II (In-RAWs), and AMP-CatB mRNA $V_{C} L N P s$ (MAC-RAWs). Among these treatments, MAC-RAWs showed the strongest bactericidal activity at all the time points tested with the percentage inhibition from $33 \%$ to $87 \%$ (Fig. 2h). When we inhibited CatB function using the CatB inhibitor II, bactericidal activity was dramatically reduced (Fig. 2h), thus suggesting the importance of releasing AMP-IB367. No significant differences in cell numbers were observed in all five groups (Supplementary Fig. 8a).

Given the potent in vitro bactericidal activity of MAC-RAWs, we tested therapeutic effects in MDRSA-induced sepsis mice with immunosuppression. After three consecutive days of cyclophosphamide (CY) treatment, decrease of body weights (BWs), white blood cells (WBCs), and lymphocytes (LYMs) mimicked an immunocompromised state in septic patients. After intraperitoneally (i.p.) infected by MDRSA, mice were treated with PBS, PBS-RAWs, or MAC-RAWs. PBS-RAWs were injected both i.p. and intravenously (i.v.) to treat local and blood bacteria. For the MAC-RAWs, we first conducted two administration methods with the same total cell number: i.p. injection alone (MAC-RAWs (i.p.)) and i.p. + i.v. injections (MAC-RAWs (i.p. + i.v.)). Since lethality in immunosuppressed sepsis is associated with ineradicable pathogens, we measured bacterial colony forming units (CFUs) in mouse blood after $24 \mathrm{~h}$ of cell transfer. Similar to PBS treatment, PBS-RAWs did not reduce bacterial burden in the blood, while MAC-RAWs (i.p.) and MAC-RAWs (i.p. + i.v.) significantly reduced bacterial CFUs in blood $(P<0.01$ and $P<0.001$, respectively) (Fig. $3 a)$. These results demonstrated bactericidal activity of MACs in vivo. Moreover, MACRAWs (i.p. + i.v.) showed much stronger ability to clear bacteria than MAC-RAWs (i.p.) (P $<0.0001$, Fig. 3a). Importantly, the survival rate of MAC-RAW (i.p. + i.v., 58\%) group was significantly improved compared to MAC-RAW (i.p.) group $(P<0.01$, Fig. 3b). Similarly, MAC-RAWs $(i . p .+i . v$. $)$ showed better therapeutic effects on survival rate $(P<0.05)$ than MAC-RAWs (i.v.) (Supplementary Fig. 8b). In the MAC-RAW (i.p. + i.v.) group, bacteria in blood were not detectable in 3 out of the 7 survived mice at $480 \mathrm{~h}$ (Fig. 3f). Then, we performed a repeat treatment for the 4 mice with persistent infections, which consequently cleared remaining bacteria in these mice (Fig. 3g). After a month, the levels of BW, WBC, and LYM of the seven survived mice fully recovered (Fig. 3c-e). Moreover, bacteria were not detectable in blood and major organs (heart, liver, spleen, lung, and kidneys) in these mice. The better therapeutic effects of MACs administrated via i.p. + i.v. injections than i.p. or i.v. injection alone may be due to the infection process of sepsis ${ }^{21,22}$. After i.p. inoculation, bacteria are transported by the lymphatic system to blood within a short period of time and then distributed to other organs via blood circulation ${ }^{21,22}$. Administration of i.p. 
$+i$.V. contributes to eliminating the bacteria in both peritoneal cavity and blood, thus leading to reduced bacterial CFUs and improved survival. When mice were $i . v$. infected by MDRSA, MAC-RAWs (i.v.) showed dramatically better therapeutic effects on survival rate $(P<0.01)$ compared to the control group (Supplementary Fig. 8c). Therefore, administration routes need to be carefully determined according to infection sites.

These promising results encouraged us to evaluate bactericidal activity using primary bonemarrow-derived macrophages (BMDMs), which are translatable for future clinical applications. We first generated BMDMs from mouse bone marrow and confirmed $\sim 83.5 \%$ cells were F4/80 positive (Supplementary Fig. 9a). Then, we screened VLNPs and tested their expression profile in BMDMs. Similar to the results in RAW264.7 cells, $V_{C} L N P s$ were more effective in mRNA delivery than other treatments (Supplementary Fig. 9b) and the maximum luminescence intensity was observed at $12 \mathrm{~h}$ (Supplementary Fig. 9c). Next, we prepared MAC-BMDMs using AMP-CatB mRNA $\mathrm{V}_{C} \mathrm{LNPs}$, and evaluated the in vitro bactericidal activity against MDRSA and multi-drug resistant Escherichia coli (MDR E.coli). Compared to BMDMs treated by PBS (PBS-BMDMs), free AMP-CatB mRNA (FrBMDMs), empty $\mathrm{V}_{\mathrm{C}} \mathrm{LNPs}$ (Em-BMDMs), and AMP-CatB mRNA $\mathrm{V}_{\mathrm{C}} \mathrm{LNPs} / \mathrm{CatB}$ inhibitor II (In-BMDMs), AMP-CatB mRNA $V_{C}$ LNPs (MAC-BMDMs) showed the strongest bactericidal activity against both MDRSA and MDR E.coli (Supplementary Fig. 9d-g). In addition, AMP-CatB mRNA $V_{C} L N P s$ did not induce obvious cytotoxicity in BMDMs (Supplementary Fig. 9h).

Next, we applied MAC-BMDMs to treat MDRSA-induced sepsis mice with immunosuppression. Based on the data from RAW264.7 cells, MAC-BMDMs were administrated to mice via both i.p. and i.v. injections. Analysis of blood at $24 \mathrm{~h}$ showed that MAC-BMDMs were more effective in eliminating bacteria than PBS-BMDMs $(P<0.001$, Supplementary Fig. 10a). Furthermore, MAC-BMDMs rescued 58\% of mice from immunosuppressed sepsis compared with only $10 \%$ rescued by PBS-BMDMs $(P<0.01$, Supplementary Fig. 10b). Except for one mouse from the MAC-BMDM group displayed persistent infection, no bacteria were found in blood in other six survived mice at $480 \mathrm{~h}$ (Supplementary Fig. 10f, g). Similarly, after a repeat treatment for that mouse, all the mice showed normal levels of BW, WBC, and LYM (Supplementary Fig. 10c-e), and undetectable levels of MDRSA in their blood and major organs.

In order to profile the biodistribution of these macrophages, we delivered mRNA encoding firefly luciferase into BMDMs (FLuc-BMDMs). Six hours post i.p. + i.v. injections of the FLuc-BMDMs, we measured the luminescence intensity in the following major organs usually infected by bacteria in this mouse mode ${ }^{21,22}$ : peritoneal cavity, spleen, liver, lung, kidneys, heart, and blood. The results showed a similar biodistribution in healthy and sepsis mice (Supplementary Fig. 10h). In the sepsis mice infected by i.p. bacterial inoculation, the rank order of luminescence intensity is as follows: peritoneal cavity, spleen, lung, liver, and blood. The BMDMs biodistribution was relatively consistent to the bacterial distribution in these sepsis mice (Supplementary Fig. 10i).

Because sepsis hosts are usually exposed to mixed bacterial infections, a formidable challenge for curing sepsis, we established a mouse sepsis model with infections by both 
MDRSA and MDR E.coli. Treatment with MAC-BMDMs significantly reduced bacterial burden in blood compared to the treatment with PBS and PBS-BMDMs, respectively (Fig. 4a). Therapeutic efficacy of MAC-BMDMs was also reflected in the survival rate (83\%), which was much higher than that of PBS group $(P<0.001)$ and PBS-BMDM group $(P<$ 0.05 ) (Fig. 4b). Finally, normal levels of BW, WBC, and LYM were observed in all survived mice (Fig. 4c-f), and no persistent infections were detected in blood and major organs.

In summary, we have developed macrophages containing antimicrobial peptides linked to cathepsin B in the lysosomes (MACs) via $\mathrm{V}_{\mathrm{C}} \mathrm{LNPs}$-mediated delivery of mRNA. Our results prove the concept that adoptive transfer of MACs beneficially reduce bacterial burden and improve survival in multi-drug resistant (MDR) bacteria-induced sepsis mice with immunosuppression by restoring innate immune defense, preventing bacterial immune evasion, and eradicating the infection. In the future, this platform will mainly focus on treating the late phase of sepsis with immunosuppression. Autologous MACs might be prepared around one week, but the weakness of patient and emergency of sepsis may make it challenging to collect and engineer autologous macrophages. Yet, with the advance of induced pluripotent stem cells (iPSCs) technologies, allogeneic "universal" macrophages may be manufactured ${ }^{23}$, enabling iPSCs-derived MACs to become an off-the-shelf therapy for clinical applications, including sepsis. Overall, the adoptive transfer of MACs may provide a strategy for curing patients with sepsis and MDR bacterial infections.

\section{Methods}

\section{Chemicals and reagents.}

The following agents were purchased from Sigma-Aldrich, including cholesterol, gentamicin, cathepsin B (CatB) inhibitor II, cyclophosphamide (CY), 5- $(N$-methyl- $N$ isopropyl) amiloride (EIPA), methyl-beta-cyclodextrin (M $\beta C D)$, and chlorpromazine hydrochloride (CPZ). The following agents were obtained from Thermo Fisher Scientific, including F4/80 monoclonal antibody, recombinant murine macrophage colony-stimulating factor (M-CSF), and LysoTracker® DND-99. 2-dioleoyl-sn-glycero-3-phosphoethanolamine (DOPE) was purchased from Avanti Polar Lipids. Bright-Glo luciferase substrate was purchased from Promega.

\section{Cells and bacteria.}

The RAW264.7 cell line was obtained from the American Type Culture Collection (ATCC) and cultured in Dulbecco's modified Eagle's medium (DMEM, ATCC) with 10\% fetal bovine serum (Gibco, Invitrogen). The cell line was not independently authenticated and mycoplasma contamination was tested by the supplier. Murine bone-marrow-derived macrophages (BMDMs) were obtained by adaptation of previous procedures ${ }^{24}$, cultured in DMEM containing $10 \%$ fetal bovine serum and $100 \mathrm{ng} / \mathrm{ml}$ M-CSF. Multi-drug resistant Staphylococcus aureus (MDRSA, ATCC BAA-44) were grown in trypticase soy agar or trypticase soy broth (BD Biosciences) at $37{ }^{\circ} \mathrm{C}$ with aeration. According to the data from ATCC, the MDRSA are resistant to the following antibiotics: amoxicillin/clavulanic acid, penicillin, ciprofloxacin, cephalothin, doxycycline, gentamicin, erythromycin, imipenem, methicillin, tetracycline, oxacillin, azithromycin, clindamycin, ceftriaxone, rifampin, 
amikacin, and tobramycin. Multi-drug resistant Escherichia coli (MDR E. coli, ATCC

BAA-2340) was grown in nutrient agar or nutrient broth (BD Biosciences) at $37{ }^{\circ} \mathrm{C}$ with aeration. According to the data from ATCC, the MDR E. coli are resistant to the following antibiotics: amoxicillin/clavulanic acid, ticarcillin, piperacillin, ampicillin/sulbactam, cefalotin, cefuroxime, cefotetan, cefpodoxime, cefotaxime, ceftizoxime, cefazolin, cefoxitin, ceftazidime, ceftriaxone, cefepime, doripenem, meropenem, ertapenem, imipenem, nalidixic acid, moxifloxacin, norfloxacin, ciprofloxacin, levofloxacin, tobramycin, aztreonam, and trimethoprim/sulfamethoxazole.

\section{Synthesis of vitamin-derived lipids.}

Compound 1, vitamin $\mathrm{B} 3$ derivative, vitamin $\mathrm{C}$ derivative, vitamin $\mathrm{D}$ derivative, vitamin $\mathrm{E}$ derivative, and vitamin $\mathrm{H}$ (also called vitamin $\mathrm{B} 7$ ) derivative, were synthesized according to the methods reported previously 25 .

\section{Preparation, characterization, and optimization of VLNP.}

mRNAs used in this study were constructed by our mRNA platform based on the reported method $^{25}$. The preparation of mRNA LNPs was reported previously ${ }^{26}$. Briefly, newly synthesized vitamin-derived lipids were formulated with DOPE, cholesterol as well as firefly luciferase (FLuc) mRNA by pipetting for in vitro screenings or by a microfluidic mixing device for ex vivo studies. The NanoZS Zetasizer (Malvern) was used to measure the size and zeta potential. The entrapment efficiency was measured by the Ribogreen assay ${ }^{26}$. The morphology of $\mathrm{V}_{\mathrm{C}}$ LNPs was examined on Thermo Scientific ${ }^{\mathrm{TM}}$ Glacios ${ }^{\mathrm{TM}}$ Cryo-TEM using the methods described before ${ }^{25}$. In order to effectively deliver mRNA into macrophages, an initial screening was performed using the five vitamin lipid nanoparticles (VLNPs), Lipofectamine 3000, and electroporation. Based on our previous method ${ }^{26}$, newly synthesized vitamin-derived lipids were formulated with DOPE, cholesterol (Lipid:DOPE:Cholesterol = 20:30:40, mole ratio) as well as FLuc mRNA (Lipid:mRNA = 10:1, mass ratio) in the initial screening. Delivery efficiency of mRNA was determined by the luciferase expression assay ${ }^{26}$. Next, the dynamics of FLuc expression followed by two rounds of optimization were carried out after the initial screening. Briefly, VcLNPs from A-1 to A-16 were prepared based on the orthogonal array design table $\mathrm{L}_{16}(4)^{4}$ and the top formulation was predicted by the FLuc expression data. After the top formulation was validated, the second round of optimization focused on fine tuning the mass ratio of $\mathrm{V}_{\mathrm{C}^{-}}$ Lipid:mRNA. Electroporation for macrophages was performed using Nucleofector kit (Lonza) and the suggested protocols by the Nucleofector $2 \mathrm{~b}$ Device.

\section{Cellular uptake and endosomal escape.}

RAW264.7 cells were seeded in a 24-well plate at $10^{5}$ cells/well and cultured for $24 \mathrm{~h}$. Then, the cells were treated by FLuc mRNA and Alexa-Fluor 647-labeled RNA (1:1, weight ratio) using Lipofectamine 3000, VcLNPs, or electroporation. After $3 \mathrm{~h}$ incubation, cellular uptake was quantified by a flow cytometer (LSRII, BD). To study endocytic pathways of VcLNPs, cellular uptake assay was performed in the presence of different endocytotic inhibitors, 5( $N$-methyl- $N$-isopropyl)amiloride (EIPA), methyl-beta-cyclodextrin (M $\beta C D)$, and chlorpromazine hydrochloride (CPZ), which inhibit macropinocytosis-, caveolae-, and clathrin-mediated endocytosis, respectively. For the endosomal escape assay, $2 \times 10^{4}$ cells 
were plated in an imaging dish (Ibidi) for $24 \mathrm{~h}$, and then $150 \mu \mathrm{g} / \mathrm{mL}$ of calcein was added to the cells with or without VcLNPs containing Alexa-Fluor 647-labeled RNA for $6 \mathrm{~h}$ at 37 ${ }^{\circ} \mathrm{C}^{27}$. After washing with PBS to remove extracellular calcein and VcLNPs, cells were lively imaged under the Nikon A1R Live Cell Imaging Confocal Microscope. The images were analysed by NIS-Elements AR 5.20.00.

\section{Analysis of lysosome co-localization.}

To test whether AMP-CatB can specificly accumulate in the lysosomes, we prepared the eGFP-CatB mRNA and delivered it into RAW264.7 cells using $\mathrm{V}_{\mathrm{C}} \mathrm{LNPs}$. Then, the lysosomes were stained with LysoTracker® Red DND-99, a well-established lysosome probe. The co-localization of eGFP-CatB and LysoTracker® Red DND-99 was visualized under the Nikon A1R Live Cell Imaging Confocal Microscope. The images were analysed by NIS-Elements AR 5.20.00.

\section{Cytotoxicity of vitamin C lipid nanoparticles in BMDMs.}

Cytotoxicity of $\mathrm{V}_{\mathrm{C}} \mathrm{LNPs}$ in BMDMs was examined by adaptation of the previous MTT assay $^{25} .2 \times 10^{4}$ BMDMs were seeded into each well of a 96-well plate in $100 \mu \mathrm{L}$ of growth medium. After $12 \mathrm{~h}$ of incubation with PBS, free AMP-CatB mRNA, empty $\mathrm{V}_{\mathrm{C}} \mathrm{LNPs}$, AMP-CatB mRNA $V_{C} L N P s / C a t B$ inhibitor II, and AMP-CatB mRNA $V_{C} L N P s$, MTT solution was added. After additional $4 \mathrm{~h}$ incubation, $100 \mathrm{uL}$ of $10 \% \mathrm{SDS}-\mathrm{HCl}$ was added into each well. The purple formazan was dissolved overnight and the absorbance was recorded at $570 \mathrm{~nm}$ by the plate reader (Molecular Devices Spectramax M5).

\section{In vitro antimicrobial assay.}

The intracellular antimicrobial assay was conducted according to the reported method ${ }^{15}$. Briefly, after treated by PBS, free AMP-CatB mRNA, empty $V_{C} L N P s$, AMP-CatB mRNA $\mathrm{V}_{\mathrm{C}} \mathrm{LNPs} / \mathrm{CatB}$ inhibitor II, and AMP-CatB mRNA $\mathrm{V}_{\mathrm{C}} \mathrm{LNPs}$, the RAW264.7 cells or BMDMs were incubated with MDRSA or MDR E.coli at multiplicity of infection of 25 for $120 \mathrm{~min}$. After washing by PBS, medium containing gentamicin $(100 \mu \mathrm{g} / \mathrm{mL})$ was supplemented and the cells were incubated for additional $1 \mathrm{~h}$ to clear extracellular bacteria. At different time points, cells were washed by PBS and lysed with $0.1 \%$ Triton-X 100 . Lastly, the lysates were cultured on the nutrient agar or trypticase soy agar for counting bacterial colony forming units (CFUs).

\section{In vivo treatment of MDR-induced sepsis mice with immunosuppression.}

All mouse experiments were performed under the protocols approved by the Institutional Animal Care and Use Committee (IACUC) of the Ohio State University and were also complied with all relevant ethical regulations as applicable. C57BL/6 mice (6-7 weeks) were purchased from the Jackson Lab. The immunosuppressed model of sepsis was conducted as the methods in the literature $21,22,28$. Briefly, C57BL/6 mice were i.p. injected $\mathrm{CY}$ at a dosage of $100 \mathrm{mg} / \mathrm{kg}$ for 3 consecutive days before bacterial infection. Their immunocompromised states were assessed by monitoring the body weights (BWs), counting white blood cells (WBCs) by a hemocytometer, and counting lymphocytes (LYMs) by the Kwik-Diff stain. For i.p. bacterial inoculation, mice were infected with $0.1 \mathrm{ml}$ of bacterial 
suspension $\left(5 \times 10^{8} \mathrm{CFUs} /\right.$ mouse for MDRSA infection and $2 \times 10^{8} \mathrm{CFUs} / \mathrm{mouse}$ for mixed MDRSA and MDR E. coli infections). For, i.v. bacterial inoculation, mice were infected with $0.1 \mathrm{ml}$ of bacterial suspension $\left(1 \times 10^{8} \mathrm{CFUs} /\right.$ mouse $)$. Afterwards, the mice were administrated by $0.2 \mathrm{~mL}$ PBS or $0.2 \mathrm{~mL}$ cell suspension (total 2 million cells/mouse). After $24 \mathrm{~h}$ infection, the blood was collected from the facial vein of live mice to quantify bacterial CFUs. The survival of mice were assessed every $12 \mathrm{~h}$ within the first 6 days and then every $24 \mathrm{~h}$ for the following 24 days based on $20 \%$ loss of BW and the early removal criteria. After 30 days, the mice were euthanized and major organs (heart, liver, spleen, lung, and kidneys) were aseptically homogenized to quantify the bacterial CFUs.

\section{Biodistribution of macrophages and bacteria.}

The biodistribution of macrophages was performed on both healthy and sepsis mice. In this experiment, we first delivered FLuc mRNA into BMDMs (FLuc-BMDMs) for $12 \mathrm{~h}$. Next, each mouse was administered $0.2 \mathrm{~mL}$ PBS or $0.2 \mathrm{~mL}$ cell suspension (total 4 million cells) via i.p. $+i$. $v$ injections. After $6 \mathrm{~h}$, bioluminescence signals in the blood, peritoneal fluid, and major organs were measured using a Xenogen IVIS imaging system as reported in the literature $^{26}$. The bacterial CFUs in the blood, peritoneal fluid, and major organs of sepsis mice induced by i.p. bacterial inoculation were quantified $6 \mathrm{~h}$ after infection.

\section{Supplementary Material}

Refer to Web version on PubMed Central for supplementary material.

\section{Acknowledgements}

We acknowledge the use of the core facility provided by the Campus Microscopy \& Imaging Facility at the Ohio State University. X.Z. acknowledges the support from Fundamental Research Funds for the Central Universities (No. DUT18RC(3)027). Y.D. acknowledges the support from National Institutes of Health (NIH) through the Maximizing Investigators' Research Award R35GM119679 of the National Institute of General Medical Sciences as well as the start-up fund from the College of Pharmacy at The Ohio State University.

\section{References}

1. Reinhart K, et al. Recognizing sepsis as a global health priority-a WHO resolution. New England Journal of Medicine 377, 414-417 (2017). [PubMed: 28658587]

2. van der Poll T Immunotherapy of sepsis. The Lancet infectious diseases 1, 165-174 (2001). [PubMed: 11871493]

3. Huttunen R \& Aittoniemi J New concepts in the pathogenesis, diagnosis and treatment of bacteremia and sepsis. Journal of Infection 63, 407-419 (2011). [PubMed: 21840338]

4. Hotchkiss RS \& Karl IE The pathophysiology and treatment of sepsis. New England Journal of Medicine 348, 138-150 (2003). [PubMed: 12519925]

5. Otto GP, et al. The late phase of sepsis is characterized by an increased microbiological burden and death rate. Critical care 15, R183 (2011). [PubMed: 21798063]

6. Hotchkiss RS, Monneret G \& Payen D Immunosuppression in sepsis: a novel understanding of the disorder and a new therapeutic approach. The Lancet infectious diseases 13, 260-268 (2013). [PubMed: 23427891]

7. Czermak BJ, et al. Protective effects of C5a blockade in sepsis. Nature medicine 5, 788 (1999).

8. Ward PA \& Fattahi F New strategies for treatment of infectious sepsis. Journal of leukocyte biology (2019). 
9. Huang X, et al. PD-1 expression by macrophages plays a pathologic role in altering microbial clearance and the innate inflammatory response to sepsis. Proceedings of the National Academy of Sciences of the United States of America 106, 6303-6308 (2009). [PubMed: 19332785]

10. Docke WD, et al. Monocyte deactivation in septic patients: restoration by IFN-gamma treatment. Nature medicine 3, 678-681 (1997).

11. Presneill JJ, Harris T, Stewart AG, Cade JF \& Wilson JW A randomized phase II trial of granulocyte-macrophage colony-stimulating factor therapy in severe sepsis with respiratory dysfunction. American journal of respiratory and critical care medicine 166, 138-143 (2002). [PubMed: 12119223]

12. Galbraith N, Walker S, Galandiuk S, Gardner S \& Polk HC Jr. The Significance and Challenges of Monocyte Impairment: For the Ill Patient and the Surgeon. Surgical infections 17, 303-312 (2016). [PubMed: 26958709]

13. Bo L, Wang F, Zhu J, Li J \& Deng X Granulocyte-colony stimulating factor (G-CSF) and granulocyte-macrophage colony stimulating factor (GM-CSF) for sepsis: a meta-analysis. Critical care (London, England) 15, R58 (2011).

14. Foster TJ Immune evasion by staphylococci. Nature reviews. Microbiology 3, 948-958 (2005). [PubMed: 16322743]

15. Garzoni C \& Kelley WL Staphylococcus aureus: new evidence for intracellular persistence. Trends in microbiology 17, 59-65 (2009). [PubMed: 19208480]

16. Lewis AJ, Richards AC \& Mulvey MA Invasion of Host Cells and Tissues by Uropathogenic Bacteria. Microbiology spectrum 4(2016).

17. Pauwels AM, Trost M, Beyaert R \& Hoffmann E Patterns, Receptors, and Signals: Regulation of Phagosome Maturation. Trends in immunology 38, 407-422 (2017). [PubMed: 28416446]

18. Giles FJ, Redman R, Yazji S \& Bellm L Iseganan $\mathrm{HCl}$ : a novel antimicrobial agent. Expert opinion on investigational drugs 11, 1161-1170 (2002). [PubMed: 12150709]

19. Linke M, Herzog V \& Brix K Trafficking of lysosomal cathepsin B-green fluorescent protein to the surface of thyroid epithelial cells involves the endosomal/lysosomal compartment. Journal of cell science 115, 4877-4889 (2002). [PubMed: 12432075]

20. Vasey PA, et al. Phase I clinical and pharmacokinetic study of PK1 [N-(2hydroxypropyl)methacrylamide copolymer doxorubicin]: first member of a new class of chemotherapeutic agents-drug-polymer conjugates. Cancer Research Campaign Phase I/II Committee. Clinical cancer research : an official journal of the American Association for Cancer Research 5, 83-94 (1999). [PubMed: 9918206]

21. Frimodt-Møller N, Knudsen J \& Espersen F The mouse peritonitis/sepsis model in Handbook of animal models of infection 127-136 (Elsevier, 1999).

22. McVicker G, et al. Clonal expansion during Staphylococcus aureus infection dynamics reveals the effect of antibiotic intervention. PLoS pathogens 10, e1003959 (2014). [PubMed: 24586163]

23. Crow D Could iPSCs Enable "Off-the-Shelf” Cell Therapy? Cell 177, 1667-1669 (2019). [PubMed: 31199910]

24. Ying W, Cheruku PS, Bazer FW, Safe SH \& Zhou B Investigation of macrophage polarization using bone marrow derived macrophages. JoVE (Journal of Visualized Experiments), (2013).

25. Zhang C, et al. Chemotherapy drugs derived nanoparticles encapsulating mRNA encoding tumor suppressor proteins to treat triple-negative breast cancer. Nano Research, 1-7.

26. Li B, et al. An orthogonal array optimization of lipid-like nanoparticles for mRNA delivery in vivo. Nano letters 15, 8099-8107 (2015). [PubMed: 26529392]

27. Su X, Fricke J, Kavanagh DG \& Irvine DJ In vitro and in vivo mRNA delivery using lipidenveloped $\mathrm{pH}$-responsive polymer nanoparticles. Molecular pharmaceutics 8, 774-787 (2011). [PubMed: 21417235]

28. Zhang L, et al. High-throughput synergy screening identifies microbial metabolites as combination agents for the treatment of fungal infections. Proceedings of the National Academy of Sciences of the United States of America 104, 4606-4611 (2007). [PubMed: 17360571] 
Vitamin lipid nanoparticle (VLNP)

Adoptive macrophage transfer

Recovery from multi-drug resistant (MDR) bacteria-induced sepsis
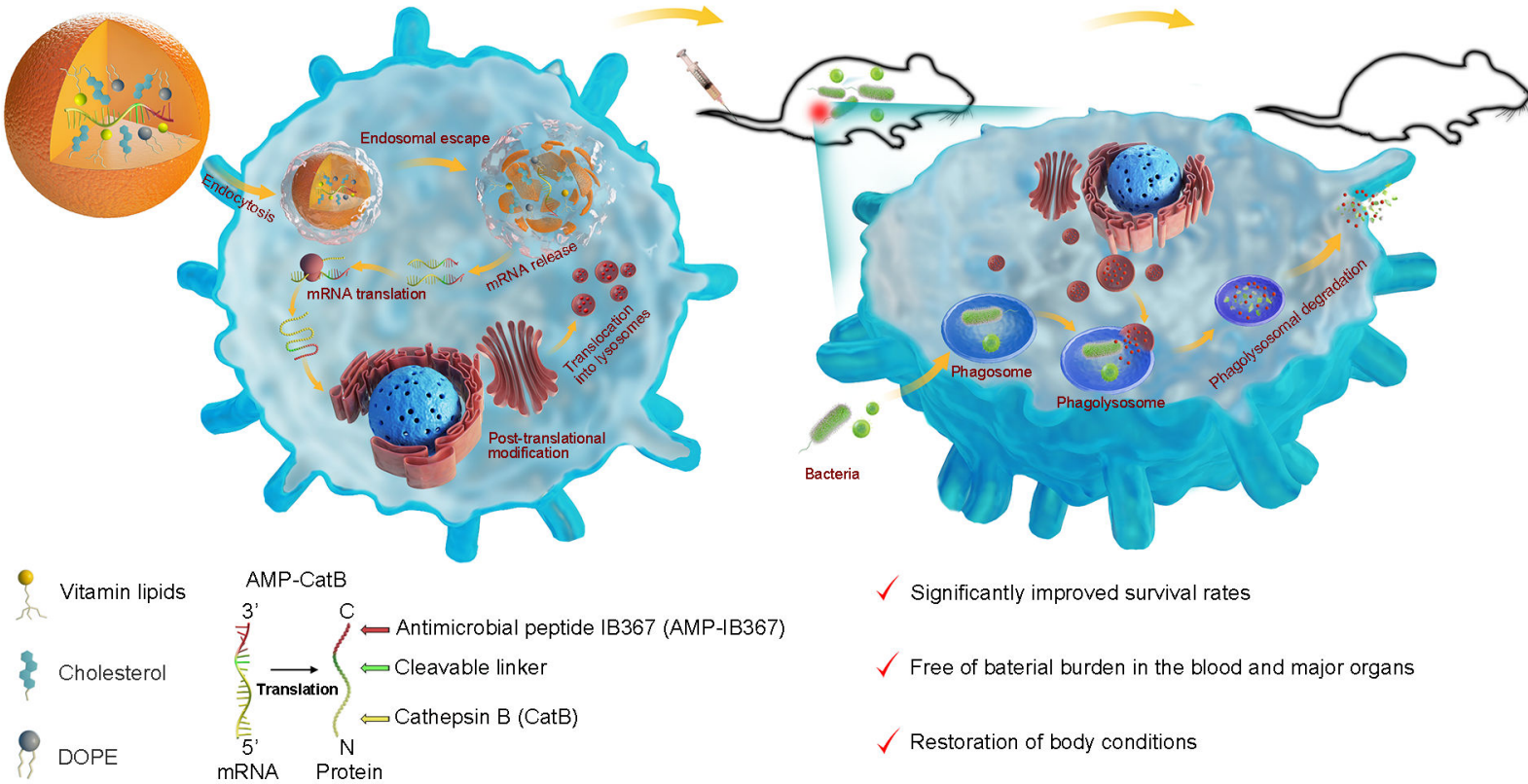

$\{$ Vitamin lipids
$\{$ Cholesterol
$\{$ DOPE

b<smiles>[R]C(=O)OCCCCCC[n+]1cccc(C(N)=O)c1</smiles>

$\mathrm{V}_{\mathrm{B} 3}$-Lipid

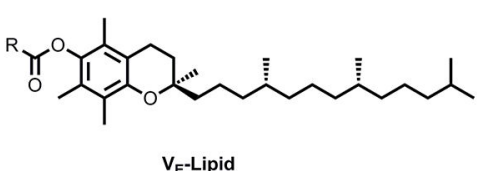

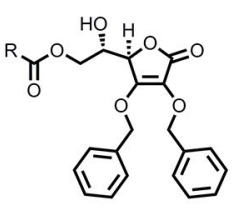

$\mathrm{V}_{\mathrm{C}}$-Lipid

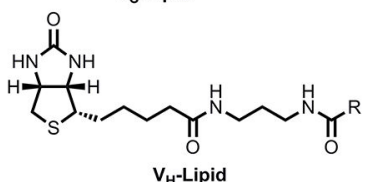

$\checkmark$ Significantly improved survival rates

$\checkmark$ Free of baterial burden in the blood and major organs

$\checkmark$ Restoration of body conditions

Fig. 1 . Schematic illustration of adoptive macrophage transfer and chemical structures of the vitamin-derived lipids.

a, Construction of MACs for sepsis therapy. MACs stands for macrophages containing antimicrobial peptides linked to cathepsin B in the lysosomes (MACs). The AMP-CatB mRNA is encapsulated in the vitamin $\mathrm{C}$ lipid nanoparticle $\left(\mathrm{V}_{\mathrm{C}} \mathrm{LNP}\right)$ and delivered to the macrophage where the mRNA is translated in the cytoplasm and translocated into the lysosomes. Within the lysosomes, the cleavable linker is cleaved by the lysosomal CatB, releasing the AMP-IB367. After phagosomes carrying MDR bacteria fuse with the lysosomes, the ingested MDR bacteria are eradicated by the pre-stored AMP-IB367. b, Chemical structures of vitamin-derived lipids including $\mathrm{V}_{\mathrm{B} 3}$-Lipid, $\mathrm{V}_{\mathrm{C}^{-}}$Lipid, $\mathrm{V}_{\mathrm{D}^{-}}$Lipid, $\mathrm{V}_{\mathrm{E}^{-}}$ Lipid, and $\mathrm{V}_{\mathrm{H}^{-}}$Lipid. 

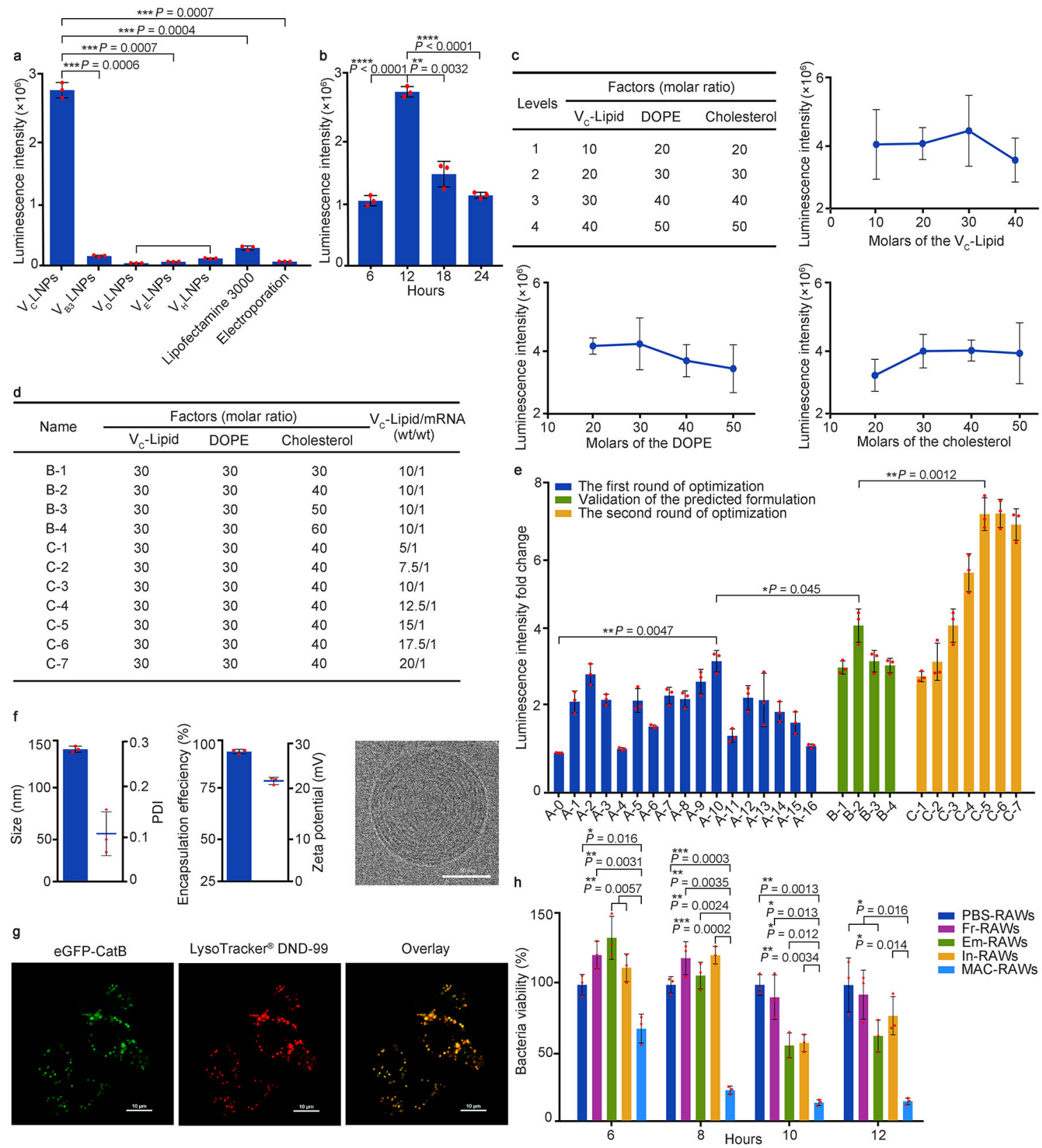

Fig. 2 |. Screening, optimization, and characterization of vitamin lipid nanoparticles (VLNPs). a, mRNA delivery efficiency of VLNPs in RAW264.7 cells. b, Expression kinetics of mRNA delivered by $V_{C} L N P s$ in RAW264.7 cells. c, The first round of optimization: four levels and impact trend of each $\mathrm{V}_{\mathrm{C}^{L}} \mathrm{LNP}$ 's component. d, Formulation table for the validation of the predicted formulation, and the second round of optimization: the mass ratio of $\mathrm{V}_{\mathrm{C}^{-}}$ Lipid:mRNA. e, Fold changes of luminescence intensity in the two rounds of optimization. $\mathbf{f}$, Characterization of the optimal $\mathrm{V}_{\mathrm{C}} \mathrm{LNP}$ formulation, including size, polydispersity index (PDI), encapsulation efficiency, zeta potential, and Cryo-TEM image (Scale bar $=50 \mathrm{~nm}) . \mathbf{g}$, Confocal imagines of the RAW264.7 cells incubated with $\mathrm{V}_{\mathrm{C}} \mathrm{LNPs}$ encapsulating eGFPCatB mRNA (Scale bar $=10 \mu \mathrm{m})$. Data in $\mathbf{g}$ are representative images from $\mathrm{n}=3$ 
independent experiments. $\mathbf{h}$, Intracellular survival of MDR Staphylococcus aureus (MDRSA) in the RAW264.7 cells. Data in $\mathbf{a}, \mathbf{b}, \mathbf{c}, \mathbf{e}$, and $\mathbf{h}$ are from $n=3$ biologically independent samples. All data are presented as mean \pm s.d. Statistical significance was analyzed by the two-tailed Student's $t$-test. $* P<0.05$, ** $P<0.01$, *** $P<0.001, * * * * P<$ 0.0001 . 

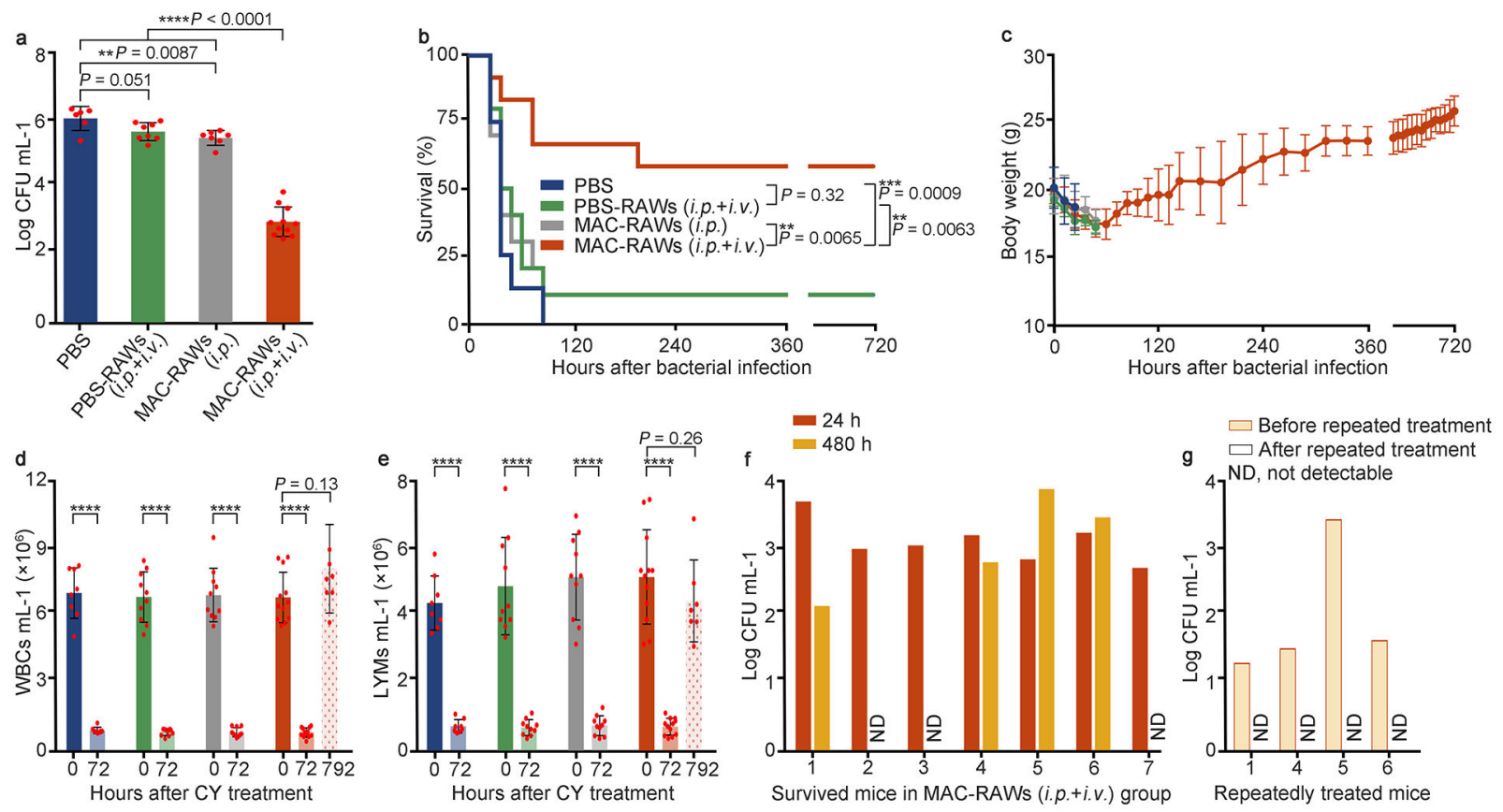

Fig. 3 |. Therapeutic effects of MAC-RAWs in MDRSA-induced sepsis mice with immunosuppression.

a, Bacterial burden in blood at $24 \mathrm{~h}$ after cell transfer. $\mathrm{n}=6,8,7$, and 11 live mice for PBS,

PBS-RAWs (i.p. + i.v.), MAC-RAWs (i.p.), and MAC-RAWs (i.p. + i.v.) groups, respectively. $\mathbf{b}$, Percentage survival of mice with sepsis induced by i.p. bacterial inoculation. c-e, The body weights (BWs), white blood cells (WBCs), and lymphocytes (LYMs) of mice. c, BWs; d, WBCs; e, LYMs. Data in b, c, d, and e (except time point $792 \mathrm{~h}$ ), $\mathrm{n}=8,10,10$, and 12 for PBS, PBS-RAWs (i.p. + i.v.), MAC-RAWs (i.p.), and MAC-RAWs (i.p. + i.v.) groups, respectively. $\mathbf{f}, \mathbf{g}$, Bacterial burden in blood of each survived mouse treated by MACRAWs (i.p. + i.v.). Data in $\mathbf{a}, \mathbf{c}, \mathbf{d}$, and $\mathbf{e}$ are the mean \pm s.d. Statistical significance was analyzed by the two-tailed Student's $t$-test. Data in $\mathbf{b}$ were analyzed by the log-rank (Mantel-Cox) test. $* P<0.05, * * P<0.01, * * * P<0.001$; ns, not significant; ND, not detectable. 

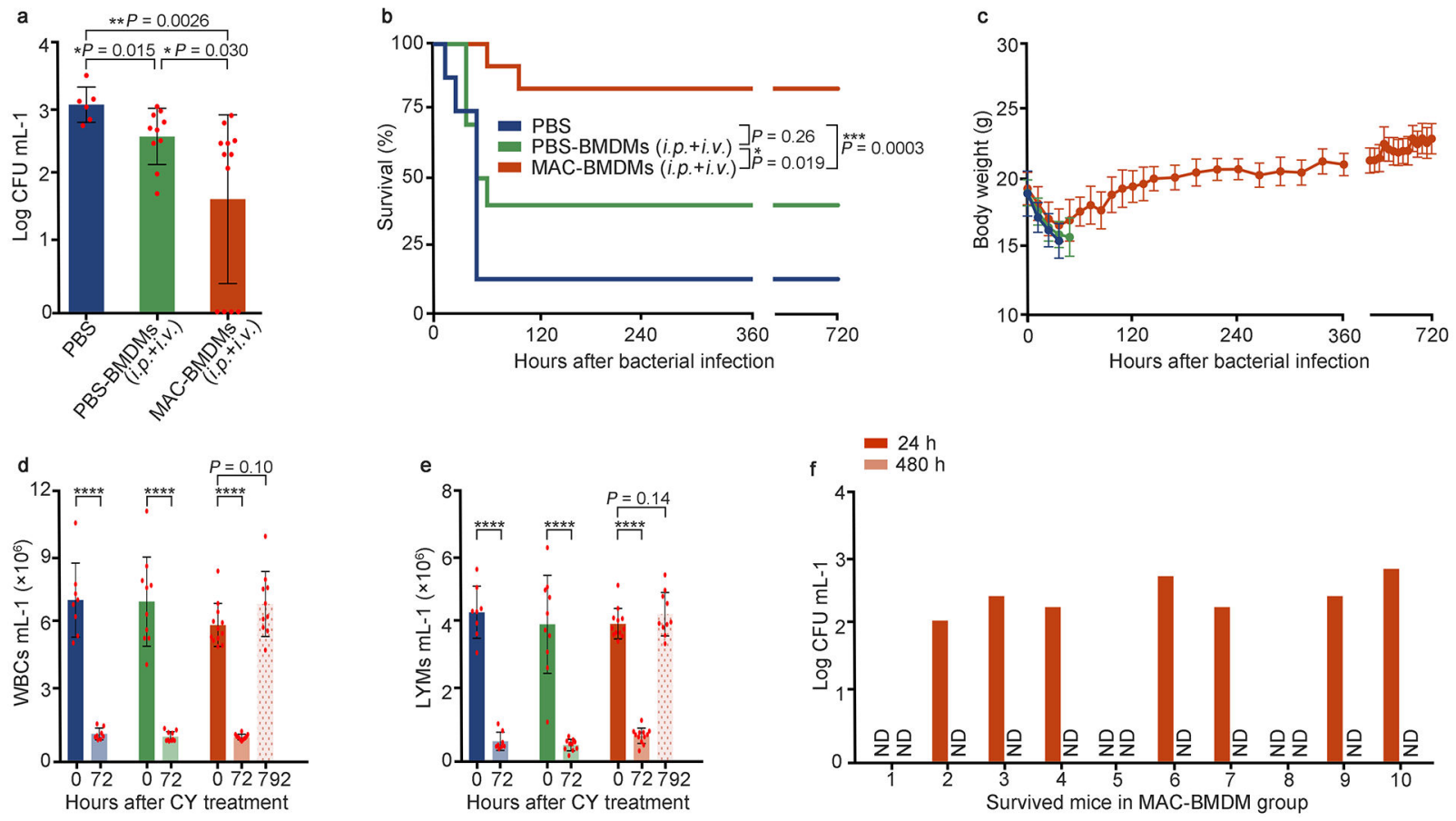

Fig. 4 |. Therapeutic effects of MAC-BMDMs in mixed MDR bacteria (Staphylococcus aureus and Escherichia coli)-induced sepsis mice with immunosuppression.

a, Bacterial burden in blood at $24 \mathrm{~h}$ after cell transfer. $\mathrm{n}=6,10$, and 12 live mice for PBS, PBS-BMDMs (i.p. + i.v.), and MAC-BMDMs (i.p. + i.v.) groups, respectively. b, Percentage survival of mice with sepsis induced by i.p. bacterial inoculation. c-e, The BWs, WBCs, and LYMs of mice. c, BWs; d, WBCs; e, LYMs. Data in b, c, d, and e (except time point 792 h), $\mathrm{n}=8,10$, and 12 for PBS, PBS-BMDMs (i.p. + i.v.), and MAC-RAWs (i.p. + i.v.) groups, respectively. $\mathbf{f}$, Bacterial burden in blood of each survived mouse treated by MAC-BMDMs. Data in $\mathbf{a}, \mathbf{c}, \mathbf{d}$, and $\mathbf{e}$ are the mean \pm s.d. Statistical significance was analyzed by the twotailed Student's $t$-test. Data in $\mathbf{b}$ were analyzed by the log-rank (Mantel-Cox) test. $* P<$ $0.05, * * P<0.01, * * * P<0.001 ;$ ns, not significant; ND, not detectable. 